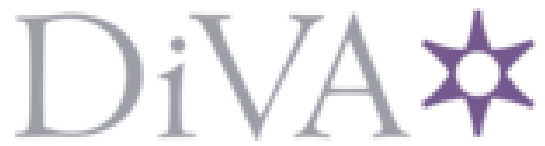

http://www.diva-portal.org

Preprint

This is the submitted version of a paper presented at 28th Annual INCOSE International Symposium.

Citation for the original published paper:

Jinzhi, L., Yuejie, W., Gürdür, D., Qi, L., Törngren, M. (2018)

MBSE Applicability Analysis in Chinese Industry

In:

N.B. When citing this work, cite the original published paper.

Permanent link to this version:

http://urn.kb.se/resolve?urn=urn:nbn:se:kth:diva-225131 


\title{
MBSE Applicability Analysis in Chinese Industry
}

\author{
Jinzhi Lu \\ KTH Royal Institute of Technology \\ inzhl@kth.se \\ Yuejie Wen \\ China Academy of Space Technology \\ wenyueiie@126.com \\ Didem Gürdür \\ KTH Royal Institute of Technology \\ dqurdur@kth.se
}

Qi Liu

Suzhou Tongyuan Software and Control Technology Co. Ltd

liuq@tongyuan.cc

\author{
Martin Törngren \\ KTH Royal Institute of Technology \\ martint@kth.se
}

Copyright (C 2018 by Jinzhi Lu, Yuejie Wen, Didem Gürdür, Qi Liu and Martin Törngren. Published and used by INCOSE with permission.

\begin{abstract}
Model-based systems engineering (MBSE) is an emerging technique widely used in current industry. It is a leading way expected to become a next generation standard practice in the systems engineering. Fundamental principles of systems engineering can be supported by a model-based approach to minimize design risks and avoid design changes in late development stages. The models can be used to formalize, analyze, design, optimize, verify and validate target products integrating engineering developments, organizations and products across domains. Though model-based development is well established in specific domains, such as software, mechanical system, electric systems, its role in integrated development from a system perspective is still a big challenge for current Chinese industry. In this paper, a survey from volunteers related with MBSE is taken by using questionnaires. The purpose of this survey is to highlight the usage and status of MBSE in current Chinese industry and address the rough understandings of MBSE concepts among system developers in China based on the answers about usages, advantages, barriers, concerns, trends of MBSE, particularly the perspective of tool-chain development.
\end{abstract}

\section{Introduction}

The purpose of this survey is a review of current usage, related concerns, obstacles and future trends of model-based systems engineering (MBSE) in various domains of Chinese industry, particularly related concerns about MBSE tool-chain development. The survey result can be used to introduce the MBSE concepts to those who are not familiar with the MBSE concepts in China. A further purpose is to propose an enhanced update report to the people who are using MBSE about the current positions of MBSE in the industry and its future trend and to provide rough clues on their future MBSE tool-chain development.

Bita (Motamedian 2013), Mary (Bone \& Cloutier 2010), Schulze (Schulze 2015), Hutchinson (Hutchinson et al. 2014) and Joseph (Elm \& Goldenson 2012) proposed a set of surveys about current MBSE applicability, industrial practices of systems engineering, model-driven engineering practices and business case for systems engineering. Though these researchers present significant findings from the surveys, much work related to more detailed contents about Chinese industries, particularly related concepts about MBSE tool-chains, still need to be done. In the existing work (Lu et al. 2017), an initial definition of MBSE tool-chain is proposed. In order to investigate further functional features and performance measurement of MBSE tool-chains, we particularly concentrate on the 
usage and more detailed techniques about MBSE tool-chains except for related contents about MBSE. This questionnaire, related to the MBSE transitioning of Chinese industry, is also an attempt to collect data in order to contribute to designing an appropriate MBSE transitioning strategy as clues.

The rest of the paper is organized as follows: Firstly, we explain the survey methodology in Section 2. In Section 3, we present the survey results in detail and evaluate the results in Section 4. Finally, we conclude the survey with a summary in Section 5.

\section{Survey Methodology}

We distributed a questionnaire, which includes 50 questions totally among different engineering groups through following social networks:

Table 1 Scopes of survey

\begin{tabular}{|l|l|c|c|}
\hline \multicolumn{1}{|c|}{ Interest group } & \multicolumn{1}{|c|}{ Domain } & \multicolumn{1}{c|}{$\begin{array}{c}\text { Potential } \\
\text { response for } \\
\text { survey }\end{array}$} & $\begin{array}{c}\text { Social } \\
\text { network }\end{array}$ \\
\hline \hline $\begin{array}{l}\text { CCOSE(http://www.ccose.org/), } \\
\text { a systems engineering interest } \\
\text { group }\end{array}$ & systems engineering & 422 & Weichat \\
\hline $\begin{array}{l}\text { Four forums about model-based } \\
\text { design }\end{array}$ & $\begin{array}{l}\text { CAD, CAE and } \\
\text { Mechanical engineering }\end{array}$ & Open & Internet \\
\hline Modelica interest group & Multi-domain modeling & 116 & Weichat \\
\hline $\begin{array}{l}\text { PDM/PLM interest group } \\
\text { Multi-domain modeling group and } \\
\text { co-simulation interest group }\end{array}$ & $\begin{array}{l}\text { CAE, Modelica and } \\
\text { co-simulation }\end{array}$ & 558 & QQ \\
\hline $\begin{array}{l}\text { Interest groups of AMESim, } \\
\text { Matlab/Simulink and Flowmaster }\end{array}$ & CAE and modeling & 998 & QQ \\
\hline $\begin{array}{l}\text { Forums of systems engineering, } \\
\text { systems engineering methodology, }\end{array}$ & Systems engineering & 1060 & Weichat \\
\hline $\begin{array}{l}\text { Forums of Forward design } \\
\text { Forums of Suzhou Tongyuan }\end{array}$ & Design method & 86 & Weichat \\
\hline
\end{tabular}

The survey is designed and developed with a set of questions administrated by a questionnaire survey website (https://www.wjx.cn/), including multiple-choice, single-choice and matrix-single-choice. Table 1 summarizes the interest group, domain, number of potential respondents, and social networks that the responses are collected from. The two platforms (QQ and Weichat) are the most popular live-chat tools in China ( $\mathrm{Wu} 2014$ ). The potential number of responses is estimated according to the size of the interest groups listed in the two platforms. 

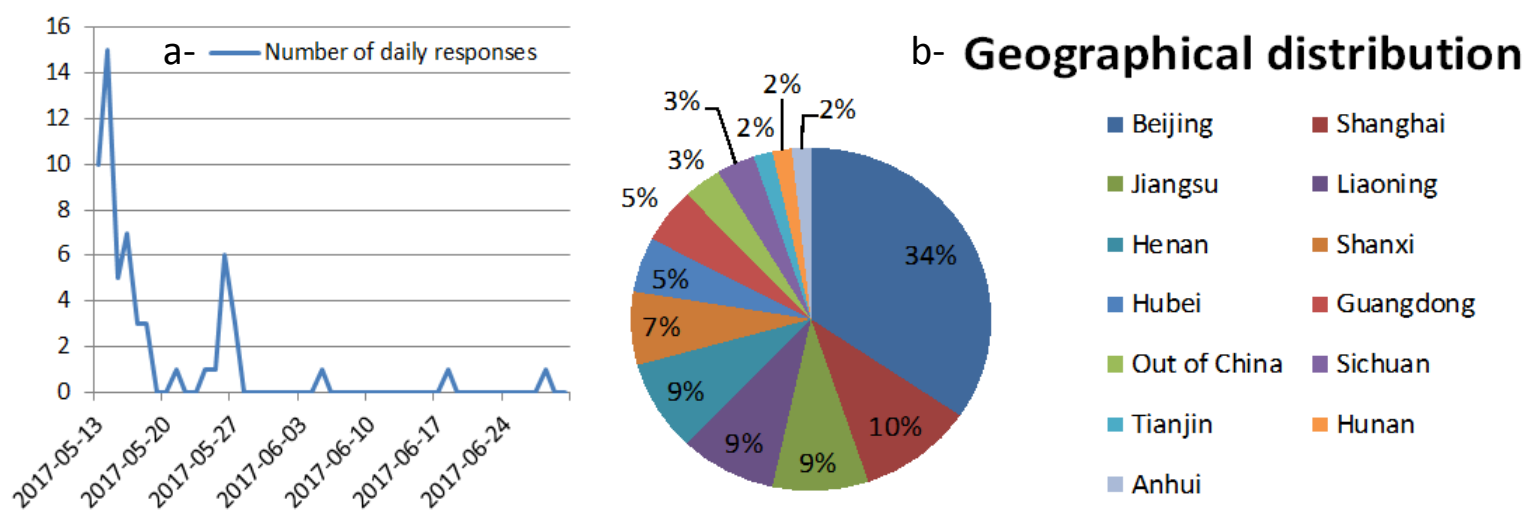

Fig. 1 a-Number of daily response; b-Graphical distribution of the responses

This survey was performed over a period of 47 days from 13th May 2017 to 30th June. We obtained 58 responses from the survey which most of the responses have been received during the first two weeks (Fig. 1-a). As shown in Fig. 1-b, roughly most respondents are from Beijing, because most research and development institutions of Chinese industries are located in Beijing.

\subsection{Respondents}

\section{Survey Results}

In order to find out what type of engineers and which positions are the most common users of MBSE, we proposed 5 questions that concentrate on the respondents' application areas, domains, roles in their groups, know-how level of MBSE and current status whether they use MBSE in their real works.

Fig. 2 illustrates the application area of the respondents (Fig. 2-a), the roles they are taking (Fig. 2-b) and domains they are working for (Fig. 2-c). Most of the responses are coming from the Aircraft industry. This can be explained by the fact that Aviation Industry Cooperation of China (AVIC) introduced MBSE and systems engineering methodology in 2013 and was launched earlier than other industries in China. Moreover, Industrial Tool Suppliers is in the second place, where Defense and Space Systems are sharing the third position.

A multi-selection question is created in order to understand which domains the respondents are belonging to. In Chinese industry, there is a trend of a new taxonomy about system design: (1) Performance analysis; (2) Structure analysis. We have added these two additional options to investigate which domains are adopting MBSE, as shown in (Fig. 2-c). From the survey results, we have found out that most of the respondents are from system design domain as expected.

In many Chinese companies, it's common that one stakeholder has different roles. Therefore, we learn respondent's roles by using a multi-selection question. Most of the respondents' role in their company is system engineer. Moreover, almost half of them are from management positions (team leaders and managers). This is especially important since the leaders or managers have better overviews about the current situations about MBSE, at least in their teams. This makes the investigation more feasible to highlight the MBSE industrial practices. 


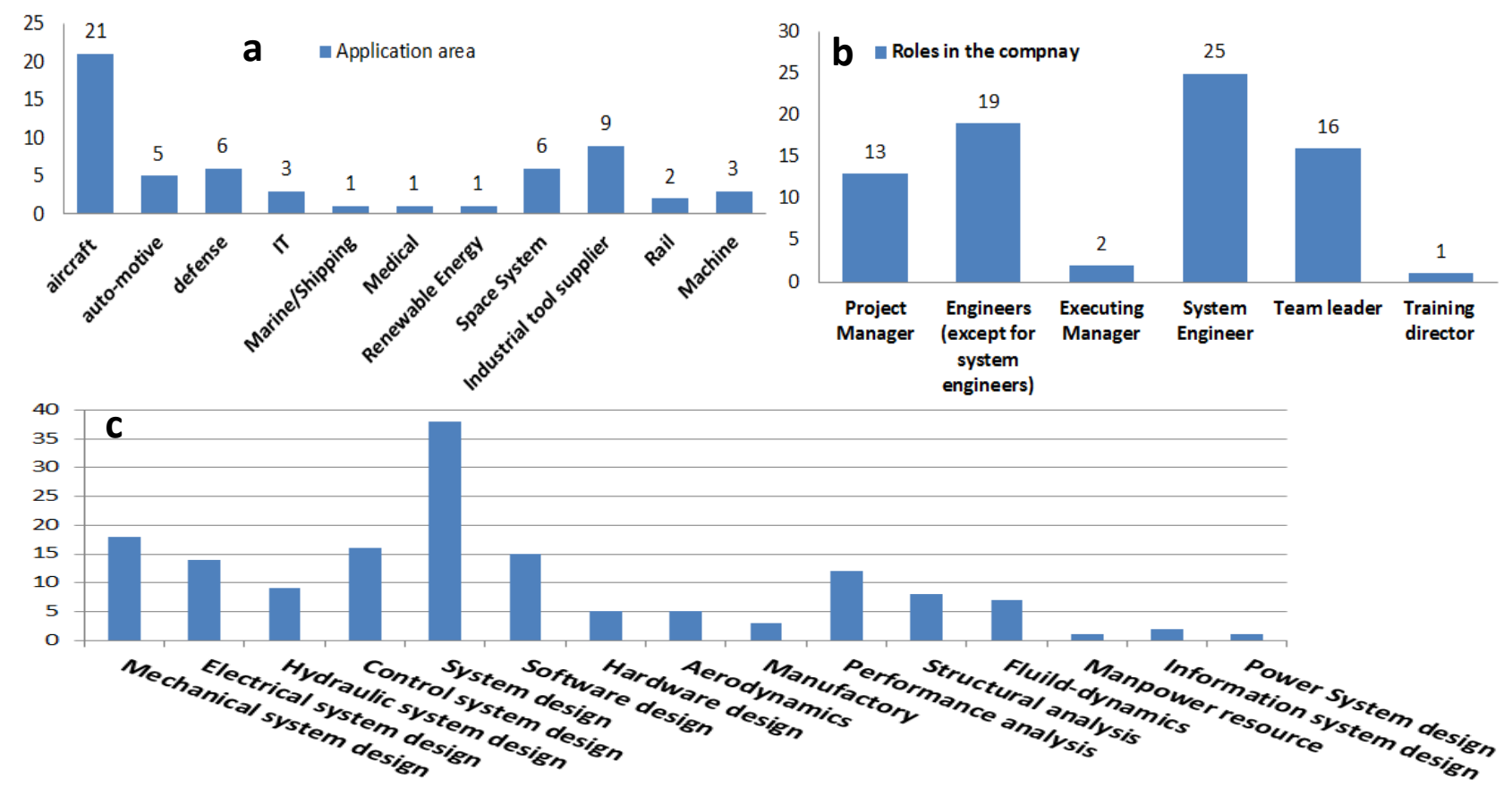

Fig. 2 a-Application domains of responses; b- roles taken by responses; c-domains responses works in

In Fig. 3, compared with the survey provided by Bita (Motamedian 2013), there are obvious gaps of know-how level of respondents (level to know MBSE) between respondents in China and ones around the whole world. The numbers of expert and advance are much less than the ones in Bita's survey (60\% for experts and $14.3 \%$ for advance right now, inferred based on results of Bita's survey). This can be explained that MBSE is an emerging concept proposed in China. Almost respondents, $55 \%$ of (32 out of 58) are using MBSE in their current jobs. Among them, almost half of them come from aircraft industries.

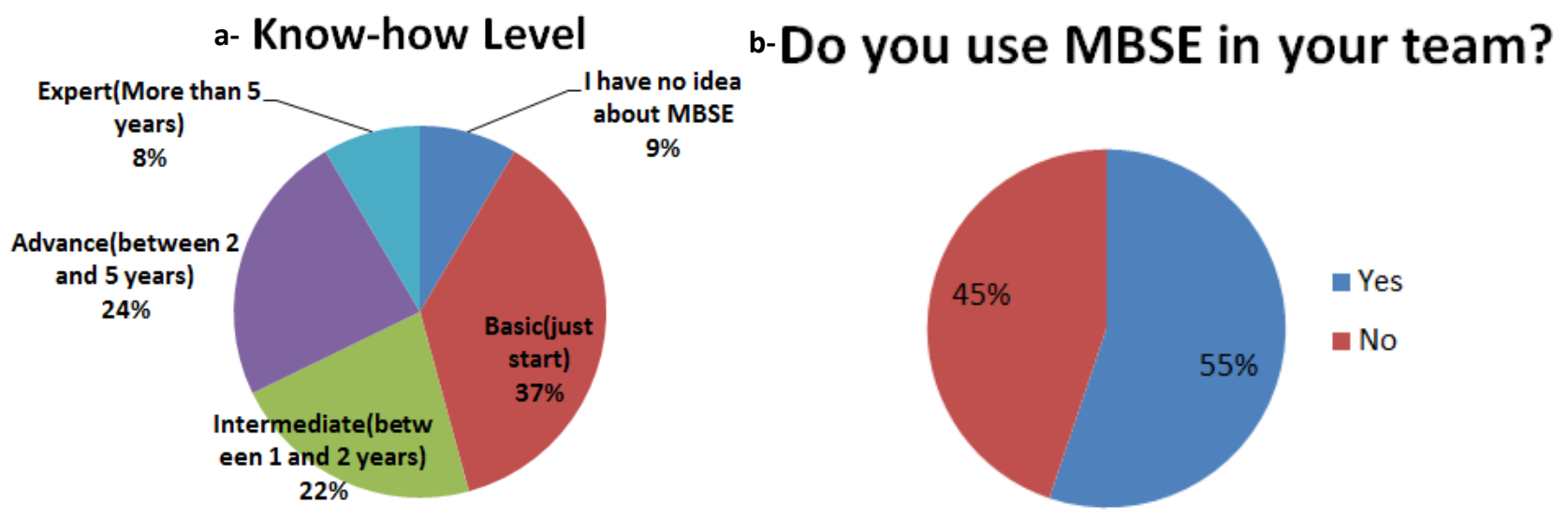

Fig. 3 a-know-how level of MBSE; b-whether MBSE is used in current work

\subsection{The extent of MBSE usage}

In this section, we investigate the extent of MBSE usage from the several perspectives, frequency using MBSE practice, usage phase of MBSE and main focus of MBSE.

In order to measure how frequently the respondents are using MBSE, we primarily classified the projects into three categories:

- Pilot project

- R\&D project

- The whole life cycle of real product development 
Moreover, we have asked questions about how frequently MBSE is used in these three types of projects when they are implemented. From Fig. 4-a, we can observe that MBSE is more widely used in pilot projects and $\mathrm{R} \& \mathrm{D}$ projects. Compared with pilot projects and R\&D projects, the frequency respondents adopt MBSE techniques in the whole life cycle of real product development project are much less. Among them, 41\% respondents do not adapt any MBSE techniques in their real product development. "Aircraft industry" seems more actively using MBSE rather than other industries. Industrial tool suppliers, "Defense“, "Space“ and "Automotive " industries also have potentials to make use of MBSE in their practices.

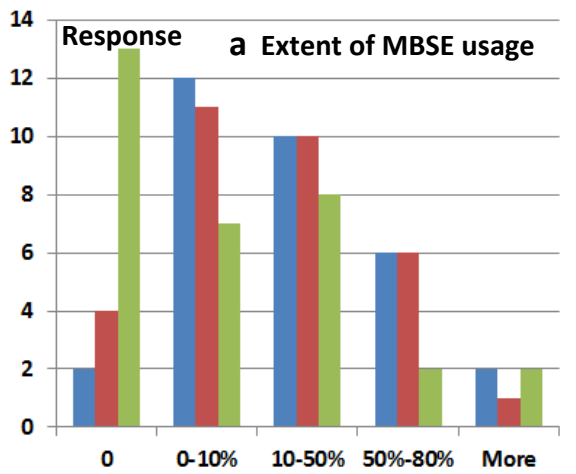

How frequently MBSE used in project than $80 \%$

$80 \%$

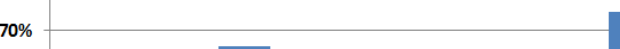

$60 \%$

$50 \%$

$40 \%$

$30 \%$

$20 \%$

$10 \%$ Project plan $\begin{aligned} & \text { Requirement } \\ & \text { development }\end{aligned}$ Verfication Validation management
Pilot project

- R\&D project

The whole life cycle of real product development b Usage phase

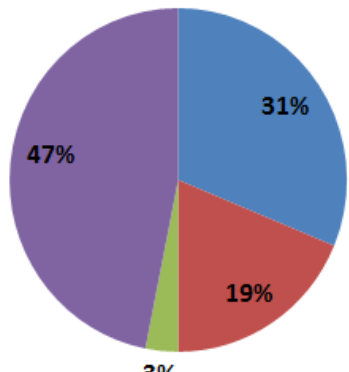

$3 \%$ pre proposal/proposal

wart of the project

Implementation of the project

Equally

Fig. 4 a-Extend of MBSE usage; b-usage phase of MBSE in the project; c-main focus of respondents on MBSE

In order to understand when respondents make use of MBSE during a project (Fig. 4-b), we define three phases:

- Pre-proposal/proposal

- The start of the project

- Implementation of the project

- Equally

Only half of the respondents use MBSE equally throughout all phases. This result has discrepancies to the definition of MBSE which is expected to cover the whole life cycle of a product. This might be explained that the MBSE concepts were introduced in China since 2013 and respondents might not understand MBSE entirely.

One question (Fig. 4-c) concentrated on the focus of MBSE usage. The question was: "Which part of MBSE does your team focus on?". According to the respondents, the top three main focuses can be listed as system architecture and system design, requirement management, and verification\& validation.

\subsection{Related Techniques about MBSE tool-chains}


To clarify the related techniques of MBSE tool-chain used in the respondents' teams, we ask questions about systems engineering, modeling techniques and integration techniques (including integrated simulation and tool-integration).

\subsubsection{Systems Engineering}

Firstly, MBSE is a model-based approach to formalize the design activities of the whole life cycle contrasted with a document-based systems engineering approach. However, as the basics of MBSE, systems engineering practices are important to MBSE practices. Therefore, we survey how system engineering standards are used in the respondents' teams. Frequency using systems engineering standards in respondents’ jobs and views used in systems engineering are also investigated.

We compared the frequency that systems engineering standards are used by the respondents who use MBSE and who do not use MBSE. As shown in Fig. 5-a, the respondents using MBSE adopt systems engineering standards more frequently than the ones who do not. Among the respondents using systems engineering standards, views (ISO/IEC 2007) used by respondents are investigated. In Fig. 5-b, architecture design, function analysis, requirement analysis are three main views concerned by respondents.
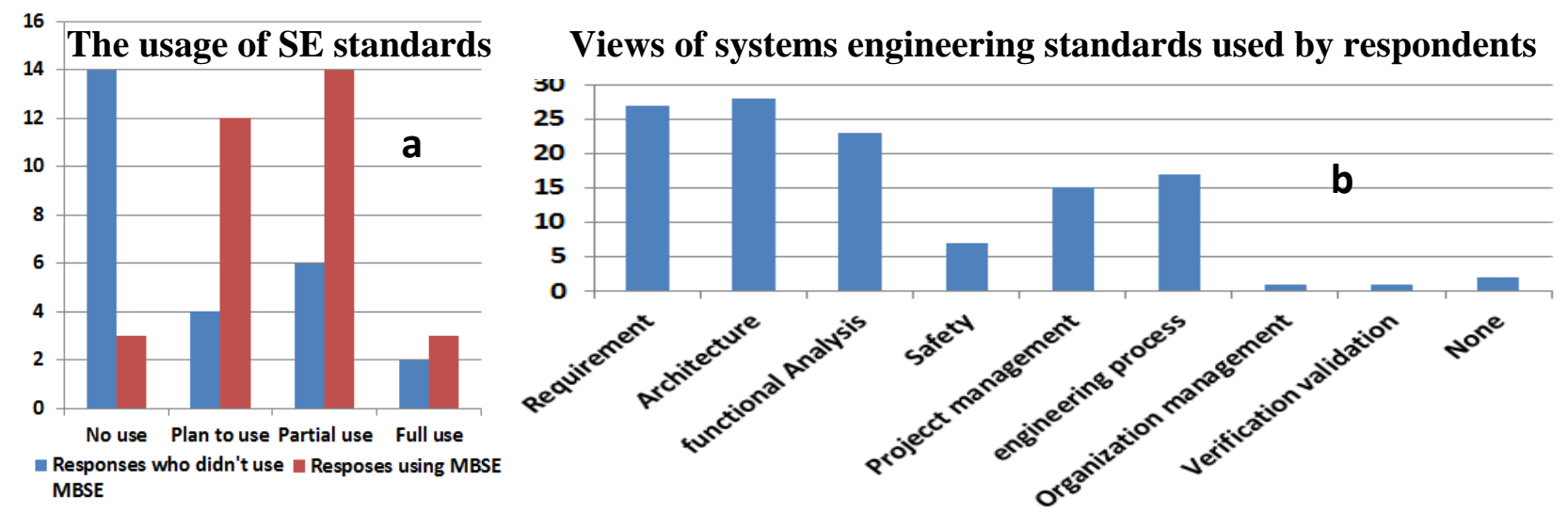

Fig. 5 a-How frequently SE standards are used; b-Views of systems engineering standards used by respondents

\subsubsection{Modeling Techniques}

In this section, we investigate modeling techniques used by respondents from seven aspects: modeling rate (the modeling frequency in respondents' work), modeling purpose, modeling language, model diagram, modeling method, modeling theory and simulation method.

We define modeling method, modeling theory and simulation method based on Saikou Diallo' encyclopedia of modeling (Diallo et al. 2015).

- Modeling method

“What is modeling?”, e.g., finite element modeling and structural equation modeling.

- Modeling theory

"How to model?", e.g., formal specification and analysis.

- Simulation method

“How to simulate?”, e.g., discrete event simulation. 

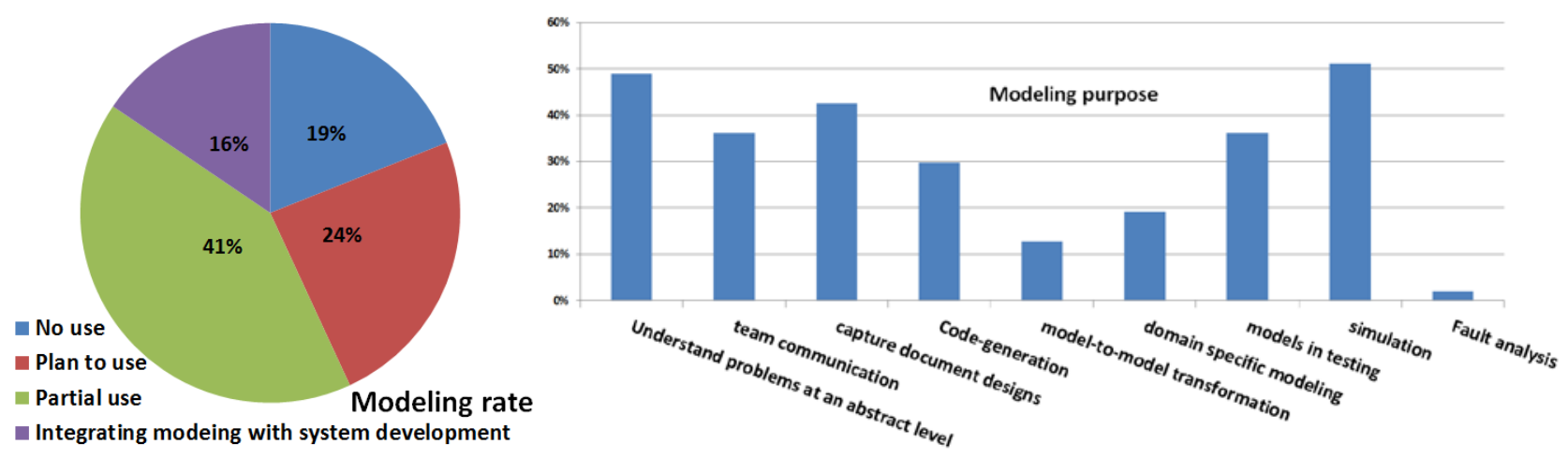

Fig. 6 Modeling rate of respondents

From the results in Fig. 6-a, almost 43\% of respondents do not use or plan to use modeling techniques. Only $16 \%$ of respondents integrate modeling with the real system development. From the result of modeling purposes in Fig. 6-b, we can find simulation and understand problems at an abstract level are top two purposes of modeling. From the analysis, we can find aircraft industry and tool suppliers are two main domains using modeling techniques.

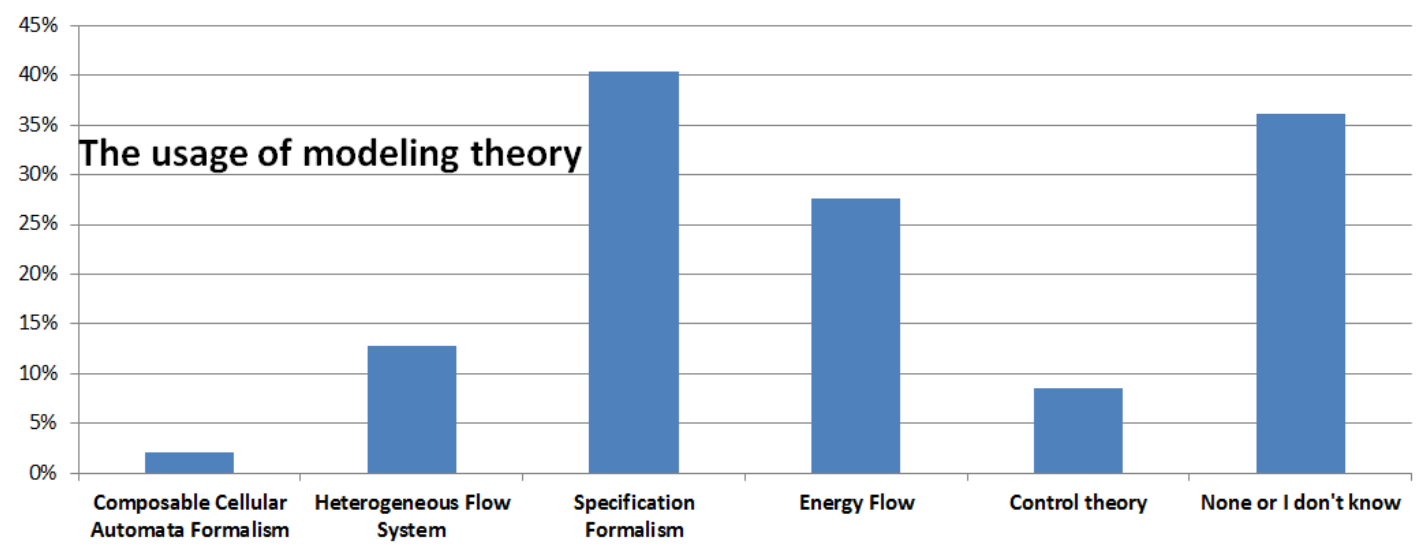

Fig. 7 Modeling theory usage

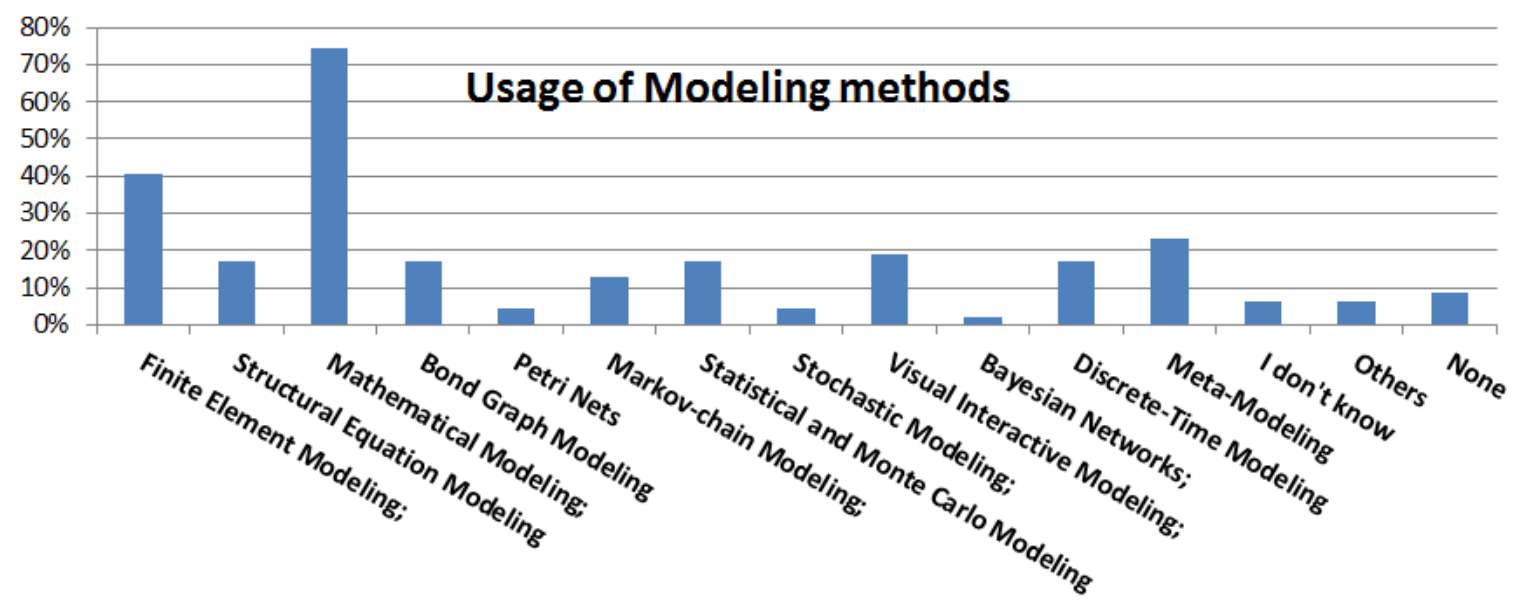

Fig. 8 Modeling method

Three aspects in (Fig. 7-Fig. 9) are used to investigate the related modeling preferences of respondents. From the results, specification formalism and energy flow theory are two main modeling theories used by respondents. Among modeling methods, mathematical modeling is the most widely used according to the survey. Moreover, we can find that numerical simulation and real-time simulation are the most widely used simulation methods in current Chinese industry. 


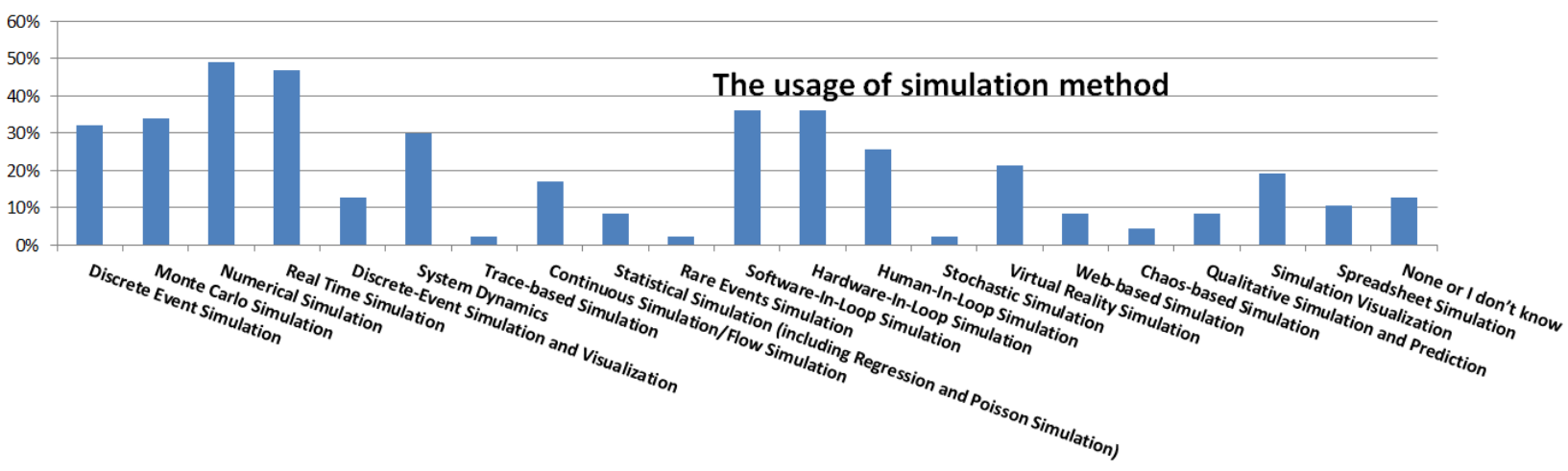

Fig. 9 The use of simulation method

More detailed questions are provided to investigate the modeling languages and language diagrams. From the survey result in Fig. 10, Matlab language and SysML are top two most widely used modeling languages. What's more, related diagrams in SysML, e.g. block definition diagram are most widely used.
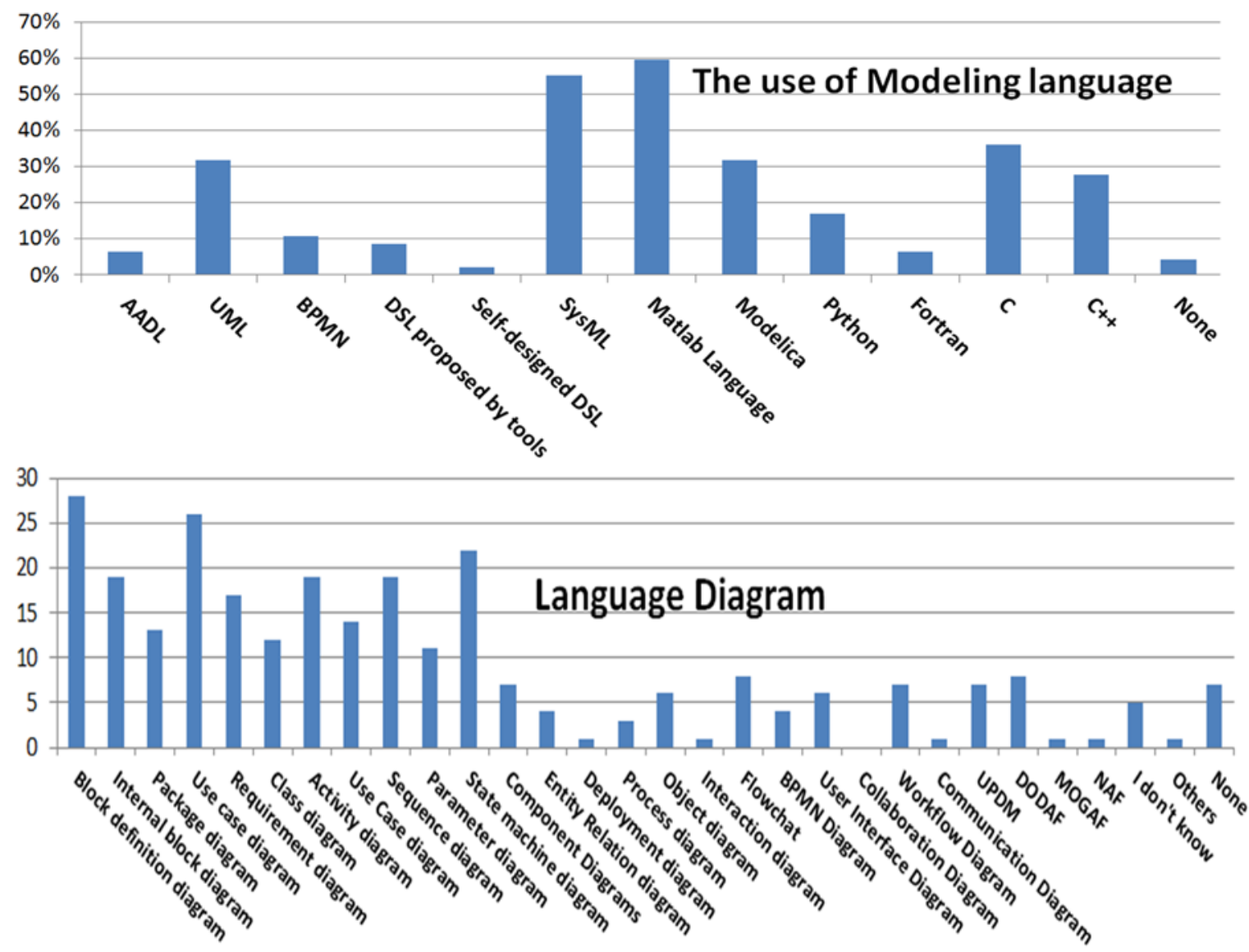

Fig. 10 The use of modeling language

\subsubsection{Techniques related to "Integration"}

In this section, we make use of several questions to investigate how the respondents implement integrations during MBSE tool-chain development, particularly in integrated simulation and tool-integration. 


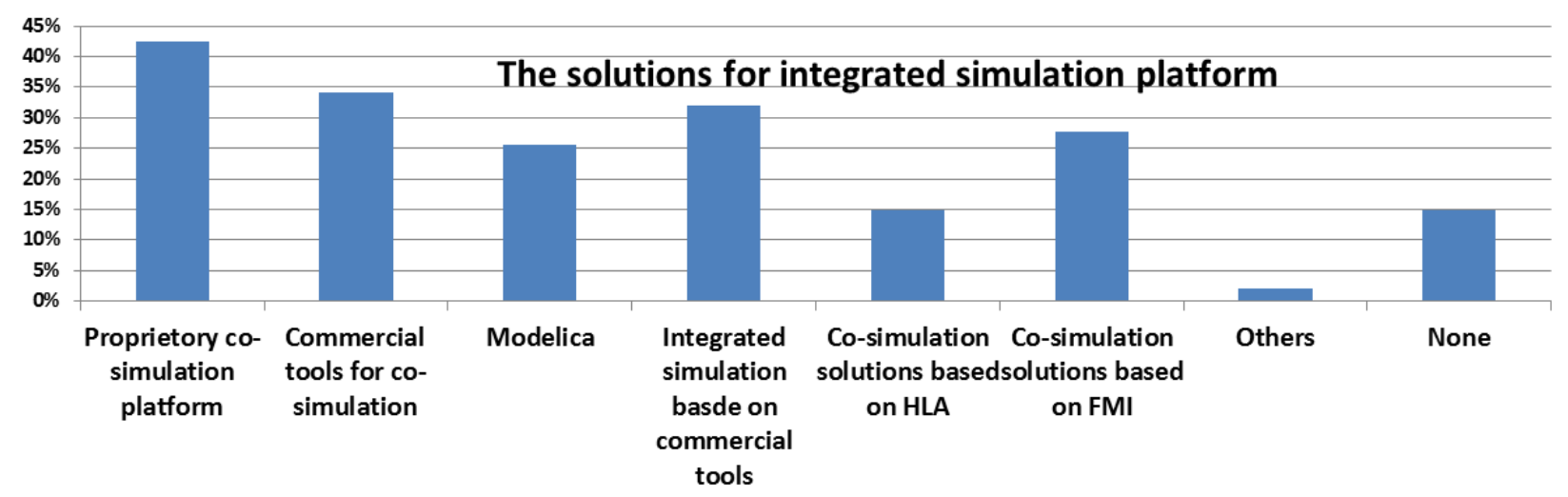

Fig. 11 Solutions for integrated simulation platform

We first concentrate on the integrated simulation platforms. High-level architecture (HLA) (Symington, Susan and Morse, Katherine L and Petty 2001), Functional Mock-up Interface (FMI) (Modelica Association Project “FMI” 2013) and Modelica (Fritzson \& Engelson 1998) are three main techniques investigated in the questionnaire. Fig. 10 shows almost $41 \%$ of respondents developed the integrated simulation platforms themselves. Many respondents make use of different techniques at the same time. Almost 26\% of respondents have used FMI as an interface standard supporting co-simulation.

During MBSE tool-chain development, tool-integration is considered as another important aspect based on our previous work. From the responses in Fig. 11-a, only 5\% of respondents did not implement tool-integration. In order to obtain more detailed contents about tool-integration, we proposed three of questions about tool-integration types, techniques used for tool-integration and metrics to measure the tool-integration performance, separately.

a The rate of tool-integration

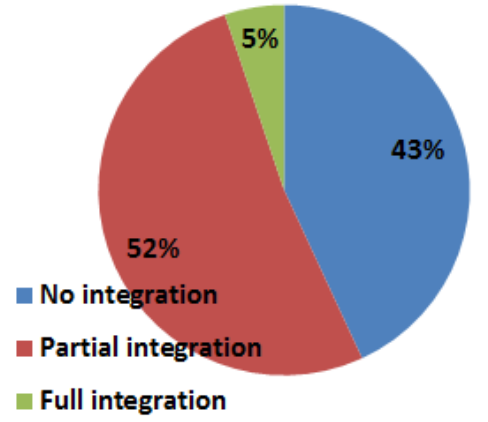

c Method for tool-integration

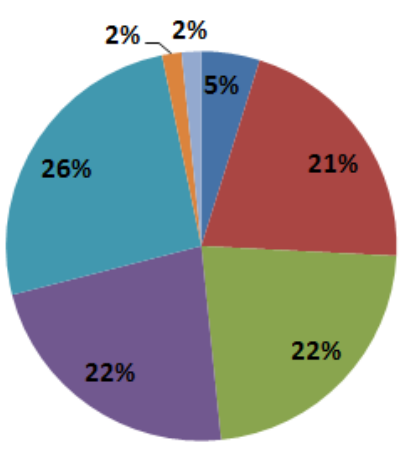

- Linked data

Rule standards oon meta-model

tools

I don't know

others b non functional properties for tool-ingration

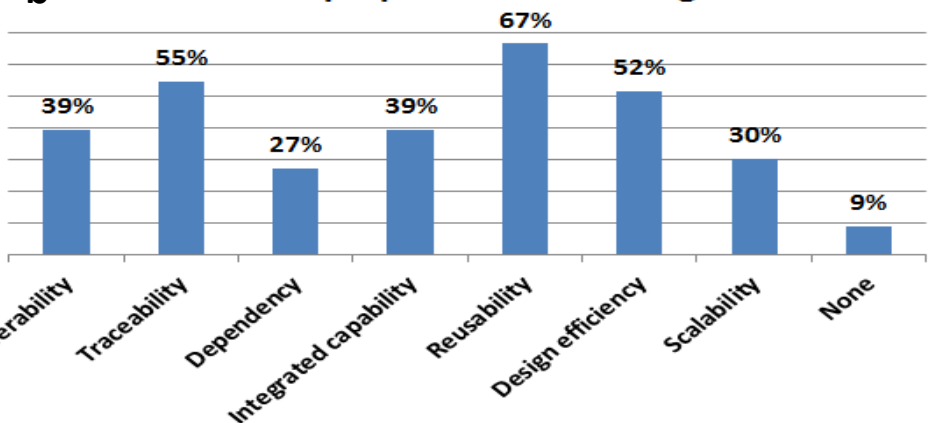

Proprietory exchange

Data exchange based on $50 \%$

Too-integration based

Based on commerical

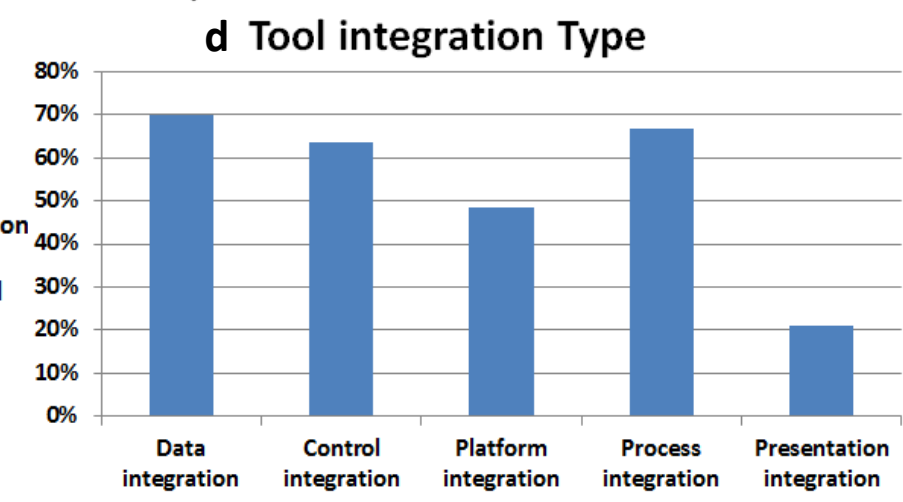

Fig. 12 a-Rate of tool-integration; b-non-functional properties for tool-integration; c Method for tool-integration; d Tool-integration Type 
Five types of tool-integration are defined to investigate how respondents implement tool-integration: (1) Data Integration (2) Control Integration (3) Platform Integration (4) Process Integration (5) Presentation Integration (Wasserman 1990). From the results in Fig. 11-d, top three types of tool-integration used for respondents' tool-chain development are data integration, process integration and control integration.

We also investigate which techniques respondents use for tool integration. From the result in Fig. 11-c, we find commercial tools to implement tool-integration is the most preference tool-integration method used in Chinese industry.

We define 7 non-functional candidate properties that can be used to assess tool-integration based on (Geraci et al. 1991):

- Interoperability

The capability of two or more components in tool-chains, e.g. tools, models can be exchanged and the capability to use the exchanged information in a heterogeneous network;

- Traceability

The capability can establish a relationship between technical recourses, system information, system development processes and social networks.

- Dependency

The capability can process the dependent relationships of technical recourses, system information, system development processes and social networks.

- Integrated capability

The capability can integrate existing tools, model and data.

- Reusability

The capability can reuse technical resources (e.g., models, data, tools) by other module or works.

- Design efficiency

The degree to which the system developers implement their design jobs by developed tool-chains.

- Scalability

The capability that tool-chain can handle a growing amount of engineering work or its potentials to be enlarged to accommodate that grow.

From results in Fig. 11-b, reusability, traceability and design efficiency are the top three most important concerns about tool-chain development.

\subsection{MBSE transitioning}

In this section, we investigate reasonable factors influence MBSE transitioning - a transitioning process implemented from current document-based development approaches to MBSE approaches. We proposed questions covering culture, economy, organization structure, personal learning and hinders.

Systems thinking has been articulated as a core competency in the practice of good systems engineering (Cloutier et al. 2015). In order to know how often respondents make use of systems thinking for their work, we proposed a question "How rate do you use systems thinking when you are doing your job?”. From results in Fig. 12, we can find only 9\% (6 out of 58) of respondents who have already used system thinking fully. Compared with other industries, the systems thinking method is more widely used in aircraft industry. Compared with other roles, system engineer and team leaders adopt system thinking more widely. 


\section{The usage of system thinking}

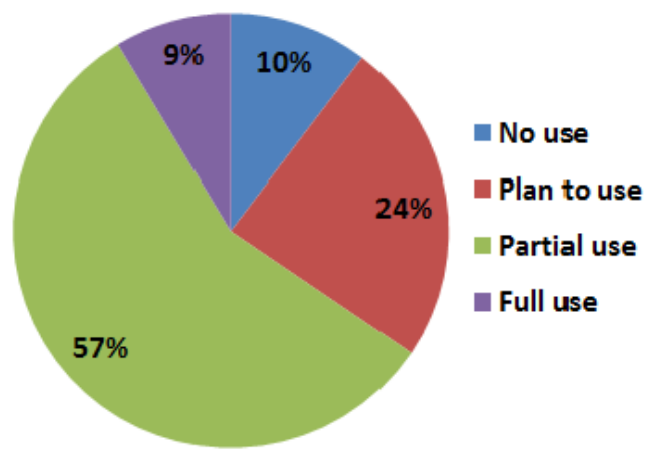

Fig. 13 The usage of system thinking

We provide a question "How much funding do you have for MBSE each year?” to investigate current funding support used for MBSE. From the result in Fig. 13-a, we can find almost half of the respondents do not have any funding for MBSE. Among industries, respondents from aircraft industry have more funding than others. The respondents that make use of MBSE can get more funding support than the ones who do not.
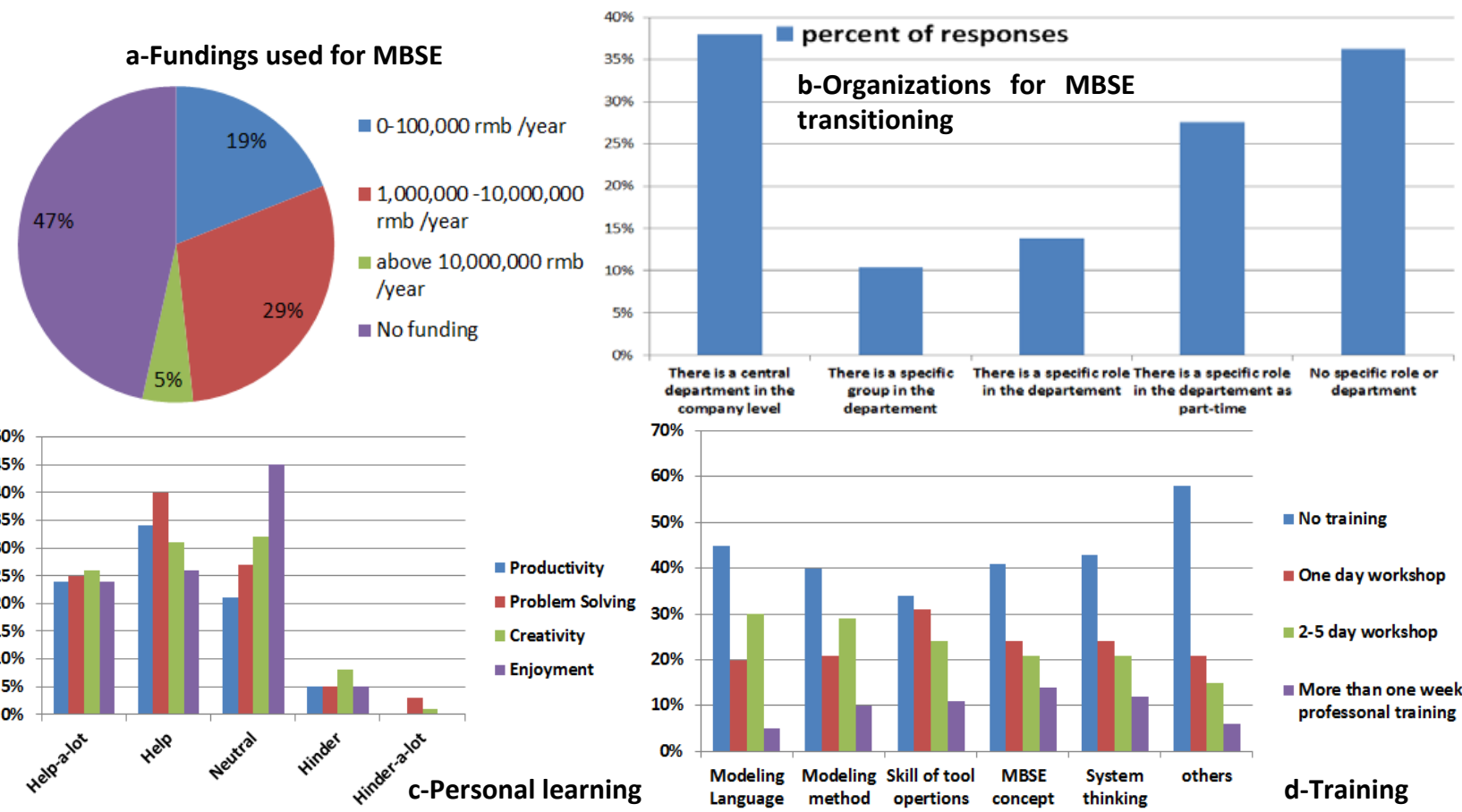

Fig. 14 a- Fundings used for MBSE; b- organizations for MBSE transitioning; c- Personal learning; dtraining

In order to understand if a centralized organization structure can help MBSE transitioning as systems engineering (Elm \& Goldenson 2012) in Chinese industries, we ask a question if they have specific organizations for MBSE transitioning. We define five types of organization structures:

- There is a centralized department for MBSE in company level

- There is a specific group for MBSE in the department

- There is a specific role for MBSE in the department

- There is a specific role for MBSE in the department as part-time

- No specific role or department for MBSE 
From the result in Fig. 13-b, respondents from the companies which have centralized departments in the company level make use of MBSE more frequently than the ones from other four organizational structures. Among industries, aircraft industry has more organizations and roles to support MBSE.

In order to investigate how MBSE influences personal learnings and promotions, we use two questions about personal learning and trainings. The result shows most of respondents think the MBSE can help their productivity, problem solving, creativity and enjoyment, as shown in Fig. 13-c. From Fig. 13-d, we can find there is a lack of related trainings of MBSE (modeling language, modeling method, skill of tool operations, MBSE concept and system thinking). Most of respondents only have one day workshop or $2-5$ day workshop. Only almost $10 \%$ of respondents (5 out of 58) have more than one week trainings.

In order to analyze obstacles of the MBSE transitioning, we make use of a multi-option to investigate which are the most serious hinders. Based on the responses in Fig. 14, the top three hinders from responses are (1) Lack of perceived value of MBSE; (2) Lack knowledge and skills of tool operations; (3) Resistance to culture change.

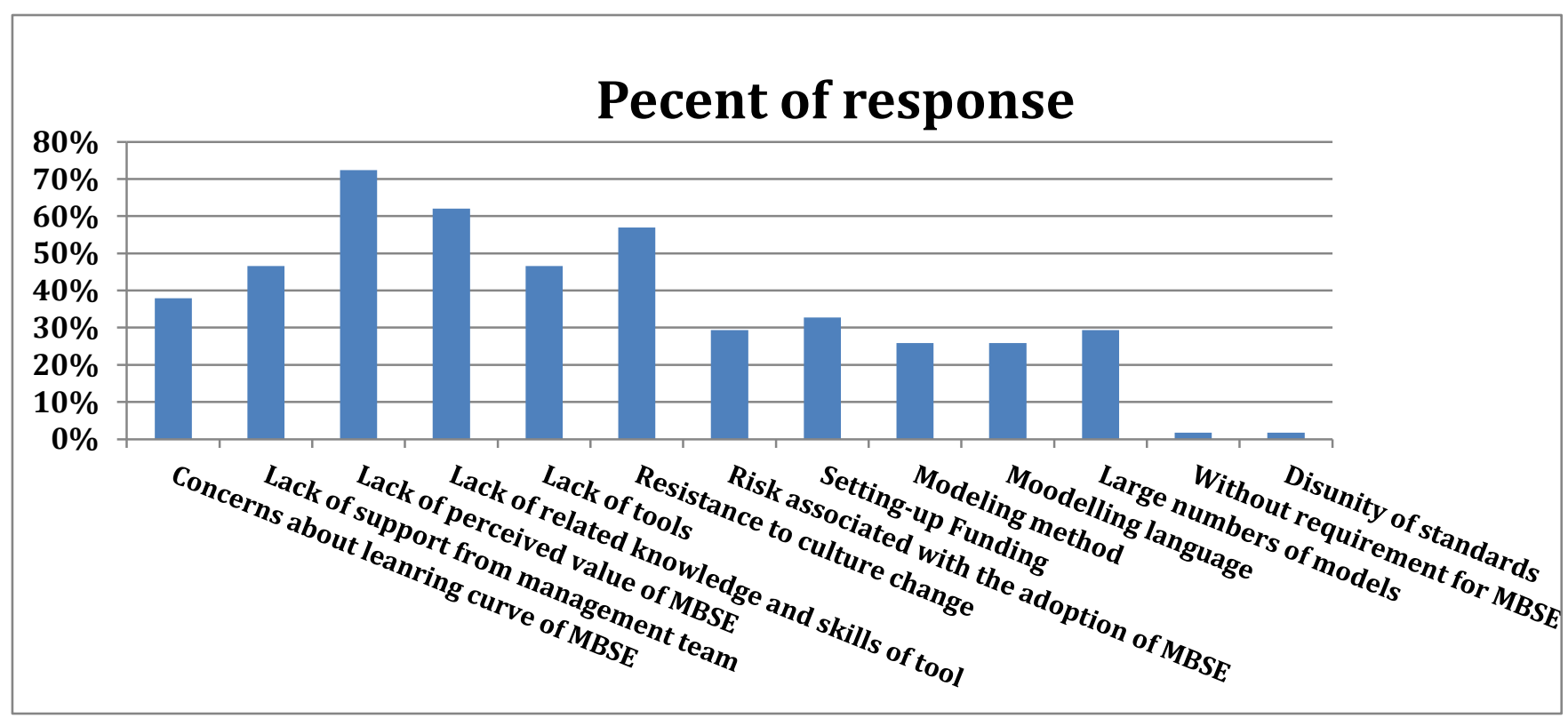

Fig. 15 Hinders of MBSE transitioning

\section{Lessons about MBSE deployment}

The findings from the responses point to a number of interesting lessons. Firstly, current MBSE industrial applications in China are implemented on some specific application areas, particularly aircraft, aerospace, vehicle, defense industries and tool suppliers. MBSE should be popularized further to other industries, for example, nuclear and marine. Respondents working for system design, especially team leaders pay more attentions to MBSE than others. This illustrates engineers, except for system engineers, need to learn more about MBSE. Compared with MBSE applicability around the whole world, MBSE industrial practices in China have just started. Currently, most of the respondents are just starting to learn related knowledges about MBSE. About half of them are not using MBSE in their work. Since 2013, the year AVIC started to popularize related concepts of MBSE, aircraft industry is increasing their interests in MBSE. This is a main reason that the respondents from aircraft industry having higher know-how level of MBSE concepts than other industries. Totally, more efforts on MBSE and its transitioning are needed for the Chinese industry.

Secondly, from results of questionnaires, we can infer that MBSE can be implemented through the whole life cycle based on most of the respondents' understandings. Current applications of MBSE are mainly focus on architecture design, requirement analysis and simulation. Further views, e.g., 
"Lities", such as dependability, should be seen as potential and important applications for future MBSE practices.

Thirdly, current Chinese industries adopt integrated simulation and tool integration to construct MBSE tool-chains. It's interesting that most of the integrated simulation platforms are developed by the respondents themselves, but most of tool integration platforms are developed based on commercial tools. No matter integrated simulation or tool integration, there are obvious shortages of adopting standards.

Fourthly, MBSE techniques are not only "SysML" and "UML". From the result, we find MBSE should include not only system modeling approach, but also virtual verification and validation. Different kinds of modeling techniques, modeling theory and modeling tools have been used in various industries. There is a need of a unified terminology to define such items.

In closing, most of the respondents think lack of understanding the value of MBSE and lack of related skills and ability are two main challenges to block MBSE transitioning processes. They think improving the capabilities of MBSE is the most important basic to MBSE transitioning. We make use a sorting question to investigate which factors are important to MBSE transitioning. The result illustrates the sequenced factors: method competency, increase SE acceptance, better definition of requirement, MBSE tool-chain and module specific product development.

This study provides useful information about the applicability of MBSE, MBSE tool-chain development and understanding of MBSE concept, yet it has several limitations. Firstly, our survey was focused on a few specific domains and stakeholders who have interests to implement MBSE in the Chinese industry. Although we covered a relative wide range of domains (e.g., PDM/PLM, model-based design, simulation, CAD, CAE) and application areas (e.g., mechanical engineering, aerospace and automotive) in China, further work to compare this particular study with existing surveys throughout the whole world is planned. Secondly, the terminologies of the current survey should be improved, such as modeling methods and simulation types.

\section{Conclusion}

Model-based systems engineering is an emerging technique aiming to replace traditional document-based systems engineering approaches. It has been proposed by the Chinese industries since 2013. More respondents have paid attentions to the related techniques. However, compared with applicability around the whole world, the Chinese industries are still relatively backward. From the results, we can conclude as follow:

- Firstly, current MBSE industrial applications in China are implemented on some specific application areas, particularly aircraft.

- Secondly, based on results of questionnaires, we can infer that MBSE should be implemented through the whole life cycle of the projects.

- Thirdly, many Chinese industries currently adopt integrated simulation and tool integration to construct their MBSE tool-chains.

- Fourthly, MBSE techniques are not only "SysML” and "UML”.

- Fifthly, during the MBSE tool-chain development, stakeholders need to consider systems engineering, modeling techniques and techniques related to integrations.

- In closing, most of the respondents think lack of understanding the value of MBSE and lack of related skills and ability are two main challenges to block MBSE transitioning.

This survey has provided a wealth of insight into current status of MBSE applicability in the Chinese industry. In the future, the survey results will be validated using more detailed interviews from industries. Comparisons with other nations will be done to show detailed shortages of current Chinese industries. A further technical report will be done to show each question, the related original data and 
more detail interviews are needed to make more specific conclusions on future MBSE transitioning. The survey results can be used as a stepping stone in designing an MBSE transitioning strategy and guidance to the future actions.

\section{Acknowledgements}

Authors are grateful for the advices and supports received from members in China Council on Systems Engineering (CCOSE). Authors recognize the exceptional contributions from forums of Systems Engineering, Suzhou Tongyuan Software and Control Technology Company, Forward design, Systems Engineering Method. Thanks for the contributions from specialists of CCOSE for the survey design, particularly Xiang Li, CEO of SysGraphLab.

\section{References}

Bone, M. \& Cloutier, R., 2010. The Current State of Model Based Systems Engineering : Results from the OMG TM SysML Request for Information 2009. 8th Conference on Systems Engineering Research, pp.225-232.

Cloutier, R. et al., 2015. Transitioning Systems Thinking to Model-Based Systems Engineering: Systemigrams to SysML Models. Ieee Transactions on Systems, Man, and Cybernetics: Systems।, 45(4), pp.662-674.

Diallo, S., Mustafee, N. \& Zacharewicz, G., 2015. Towards an encyclopedia of Modeling and Simulation methodology. In Intergovernmental Panel on Climate Change, ed. 2015 Winter Simulation Conference (WSC). IEEE, pp. 2692-2703.

Elm, J.P. \& Goldenson, D.R., 2012. The Business Case for Systems Engineering Study : Results of the Systems Engineering Effectiveness Survey,

Fritzson, P. \& Engelson, V., 1998. Modelica - A unified object-oriented language for system modeling and simulation. In Proceedings of the 12th European Conference on Object-Oriented Programming (ECOOP '98). pp. 67-90.

Geraci, A. et al., 1991. IEEE Standard Computer Dictionary. A Compilation of IEEE Standard Computer Glossaries,

Hutchinson, J., Whittle, J. \& Rouncefield, M., 2014. Model-driven engineering practices in industry: Social, organizational and managerial factors that lead to success or failure. Science of Computer Programming, 89(PART B), pp.144-161.

ISO/IEC, 2007. Systems and software engineering - Recommended practice for architectural description of software-intensive systems,

Lu, J. et al., 2017. An Investigation of Functionalities of Future Tool-chain for Aerospace Industry. INCOSE International Symposium, 27(IS), pp.1408-1422.

Modelica Association Project “FMI,” 2013. Functional Mock-up Interface for Model Exchange and Co-Simulation,

Motamedian, B., 2013. MBSE Applicability Analysis. International Journal of Scientific \& Engineering Research, 4(2), pp.1-7.

Schulze, S.-O., 2015. SYSTEMS ENGINEERING in industrial practice. , pp.1-67.

Symington, Susan and Morse, Katherine L and Petty, K., 2001. IEEE Standard for Modeling and Simulation (M\\&S) High Level Architecture (HLA)-Federate Interface Specification (IEEE Std 1516-2000).

Wasserman, A.I., 1990. Tool Integration in Software Engineering Environments. Software Engineering Environments, 467, pp.137-149.

Wu, J., 2014. From Wechat To We Fight: Tencent and China Mobile’S Dilemma. 


\section{Biography}
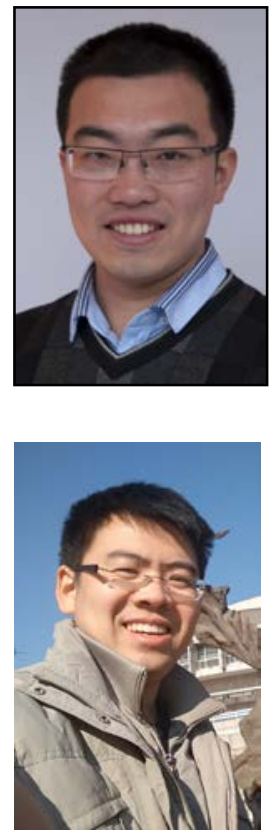

Yuejie Wen is a senior engineer of Shenzhou Institute, China Academy of Space Technology (CAST), and a co-founder of China Council on Systems Engineering (CCOSE). He is also the general secretary of Digital Design \& Manufacturing Committee affiliated to China Graphics Society (CGS). He interests in systems engineering, knowledge management, design/dependency structure matrix, and contributes to boost the scholar community of systems engineering in China.

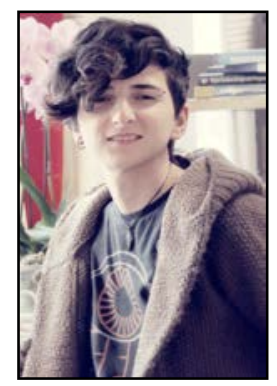

Didem Gürdür received her B.Sc. degree in Computer Engineering and M.Sc. degree from Sustainable Environment and Energy Systems program. Currently, she is a Ph.D. student at KTH Royal Institute of Technology, Mechatronics Division, in the research area of Integrated Engineering Environments. Her current research interest includes interoperability assessment, data visualizations, visual analytics, knowledge discovery, tool integration and interoperability in Cyber-physical Systems.

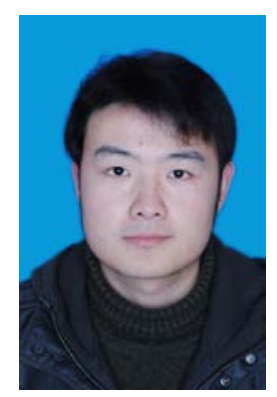

Qi Liu, the CTO of Suzhou Tongyuan Software and Control Technology Company. He and his team developed a series of model based system design, simulation and optimization tools. His current research interest includes model based system engineering, system modeling and simulation, co-simulation and MBSE enterprise transitioning.

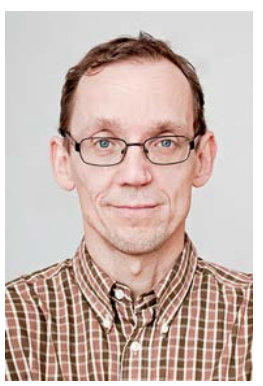

Martin Törngren has been a Professor in Embedded Control Systems at the Mechatronics division of the KTH Department of Machine Design since 2002. He has particular interest in Cyber-Physical Systems, model based engineering, architectural design, systems integration, and co-design of control applications and embedded systems. He has authored/co-authored more than 100 peer reviewed publications, and also been in charge of developing and leading graduate and continued education courses. He spent time as a post-doc at the EU-JRC, and did a 10 month sabbatical 2011/12 at UC Berkeley. 